\title{
Industrial interference and radio astronomy
}

\author{
A. Jessner \\ Max-Planck-Institut fuer Radioastronomie, Auf dem Huegel 69, 53121 Bonn, Germany \\ Correspondence to: A. Jessner (jessner@mpifr-bonn.mpg.de)
}

\begin{abstract}
The interferer - victim scenario is described for the case of industrial interference affecting radio astronomical observatories. The sensitivity of radio astronomical receivers and their interference limits are outlined. EMC above $30 \mathrm{MHz}$ is a serious problem for Radio Astronomy. Interferer (CISPR) and victim (ITU-R RA 769) standards are not harmonised. The emissions from the interferer and their spectral characteristics are not defined sufficiently well by CISPR standards. The required minimum coupling losses (MCL) between an industrial device and radio astronomical antenna depends on device properties but is shown to exceed $140 \mathrm{~dB}$ in most cases. Spatial separation of a few $\mathrm{km}$ is insufficient on its own, the terrain must shield $>30-40 \mathrm{~dB}$, additional mitigations such as extra shielding or suppression of high frequency emissions may be necessary. A case by case compatibility analysis and tailored EMC measures are required for individual installations. Aggregation of many weak rfi emitters can become serious problem. If deployment densities are high enough, the emission constraints can even exceed those for a single interferer at a short distance from the radio observatory. Compatibility studies must account not only for the single interferer but also for many widely distributed interference sources.
\end{abstract}

\section{The victim}

Radio astronomy installations (observatories) are a largescale and cost intensive part of the scientific infrastructure that is fully financed by the tax payer. They have to be large and expensive, because the radio signals we receive from cosmic sources are extremely attenuated and one needs large antennas with large collecting areas and sophisticated cryogenic receivers for their detection. Radio astronomy belongs to the realm of fundamental science. But it has also led to quite a few technical applications (e.g. feed horns for satellite dishes), and its techniques are used in vital measurements such as the establishment of a very precise global coordi- nate system which even accounts for the motion of the continents but these have been serendipitous side effects, which are nevertheless welcome by all. The aim of radio astronomical research is however to understand the structure of the cosmos we find ourselves in, the development of the universe, its galaxies, stellar systems and planets as well as the very structure of space-time itself. Radio astronomy also plays a significant role in the education of new scientists and engineers in astrophysics as well as in the fields of radio engineering, image processing, data acquisition and many more. As such it is a long-term investment of society into its own future, something outside the scope of commercial interests that demand an identifiable return within a predictable timescale. It is only logical that governments try to protect their investments like anyone else and perhaps for that reason, radio astronomy became a recognised radio service within the radio regulations (RR) of the $\mathrm{ITU}^{1}$ (Radio Regulations, 2012), a global set of rules governing the allocation and use of radio spectrum which is updated regularly by the world radio conferences. Radio astronomy is a passive (listen only) radio service detecting natural radio emissions in contrast to most other usage of the radio spectrum that involves technical radio transmitters (active services).

One of the reasons for the unequalled sensitivity of radio astronomical measurements lies in the principle of comparing the received radio flux density from an unknown source to that of the know radio background (Dicke, 1946), whereby sensitivities of more than $50 \mathrm{~dB}$ below the background noise $(S / N<-50 \mathrm{~dB})$ can be achieved. Such sensitive radiometric measurements are only possible, if the reference sky (background) is free from interference itself, and that to a degree exceeding the most sensitive measurements. As a result, radio astronomy has been allocated specific protected bands, mostly around frequencies where molecules of the tenuous cosmic gases emit their spectral lines (Fig. 1).

\footnotetext{
${ }^{1}$ ITU: International Telecommunication Union (Geneva)
} 


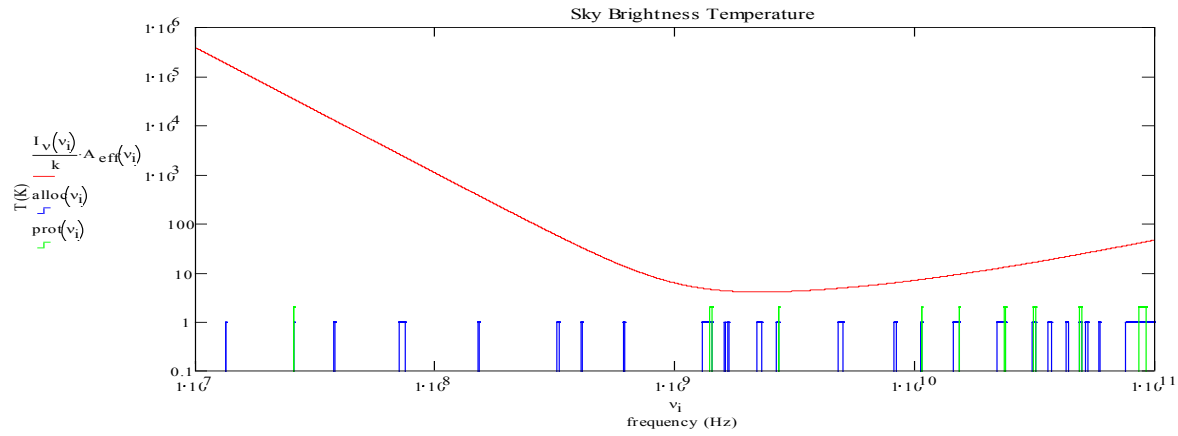

Fig. 1. The Radio sky temperature (Cane, 1979; Ellington, 2005) and radio astronomical allocations between $10 \mathrm{MHz}$ and $100 \mathrm{GHz}$. Red: Sky temperature for the Zenith, green: passive bands allocated to radio astronomy, blue: bands shared with other services.

Radio astronomy has an exclusive use of only $0.7 \%$ of the radio spectrum below $30 \mathrm{GHz}$, the majority of the allocated bands are shared with other radio services. The choice of these bands is not free and neither is that of the interference thresholds. Both are determined by the physics of the distant emitter and the physical limits of a radio receiver and its use in sensitive radio astronomical measurements. Exclusive bands for radio astronomy are covered by the RR Footnote 5.340 (Radio Regulations, 2012) which says that "no emissions are permitted in a list of bands" with emissions being termed as radiation coming from a radio station (of any kind) as the result of its active operation. Radiation from other parts of the station, e.g. the local oscillator or the IF of a receiver is not an emission in that sense, an important distinction as we will see later on.

The ITU Recommendation RA 769 (ITU-R, 2003) lists the specific threshold levels of radio interference for which the interference becomes detrimental to the operation of the radio observatory. These levels are specified as $10 \mathrm{~dB}$ below the radiometric receiver sensitivity, typically an $\mathrm{I} / \mathrm{N}$ of $-60 \mathrm{~dB}$, implying that measurement results should not be affected by an additional error of more than $10 \%$.

Radio astronomical antennas have forward gains depending on frequency, but typically of the order of $50-60 \mathrm{dBi}$ within a narrow beam of a width of typically a few minutes of arc. Interference enters the receiver mainly via sidelobes with varying degrees of gain or attenuation. For interference estimates the radio astronomical receiver is assumed to have an average gain of $0 \mathrm{dBi}$ for reception from random directions. Measurement errors of radiometric noise power measurements decrease with the square root of the product of band width and averaging time (Dicke, 1946) requiring an appropriate scaling of interference thresholds. As a result of these considerations, the interference thresholds are given by Eq. (1)

$S_{H}=0.1 \cdot \frac{4 \pi k f^{2} \cdot\left(T_{\mathrm{ant}}+T_{\mathrm{rec}}\right)}{c^{2} \sqrt{\Delta f \tau}}$

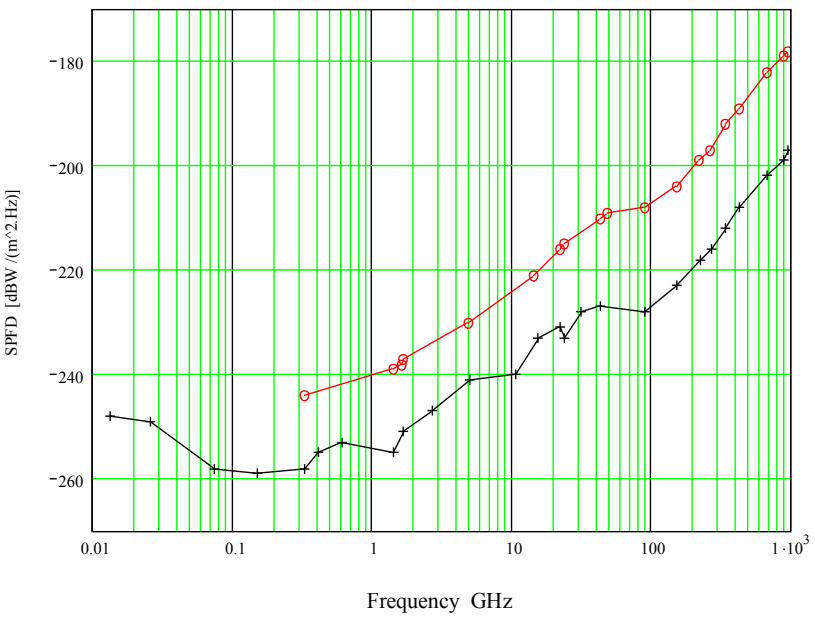

Fig. 2. Detrimental spectral flux density values for radio astronomy according to ITU-R RA 769. Black: continuum limit, red: spectroscopic limit.

taken from ITU-R RA 769. As usual, $k$ is the Boltzmann constant and $c$ the speed of light. The result $S_{H}$ is the spectral flux density threshold ( $\mathrm{W} \mathrm{m}^{-2} \mathrm{~Hz}^{-1}$ ), with $f$ being the centre frequency of the band, $T_{\text {ant }}$ the typical antenna temperature and $T_{\text {rec }}$ the typical receiver temperature, $\Delta f$ is the measurement bandwidth and $\tau$ the duration of the signal integration. ITU-R RA 769 lists these quantities in its Tables 1 and 2 for continuum (broad band) and spectroscopy (narrow band) measurements. Figure 2 shows the thresholds as a function of frequency for an integration time of $2000 \mathrm{~s}$.

The interference limits are defined for the victim in the case of radio astronomy as averages over time and bandwidth.

Astronomical sources are weak and therefore the astronomical unit of spectral flux density is 1 Jansky $(\mathrm{Jy})=10^{-26}$ $\mathrm{W} \mathrm{m}^{-2} \mathrm{~Hz}^{-1}$, named after Karl Jansky (1905-1950), the discoverer of cosmic radio waves. The interference thresholds are of the order of $1 \mathrm{Jy}$ in UHF and L-band and rising roughly proportional to the observation frequency. 

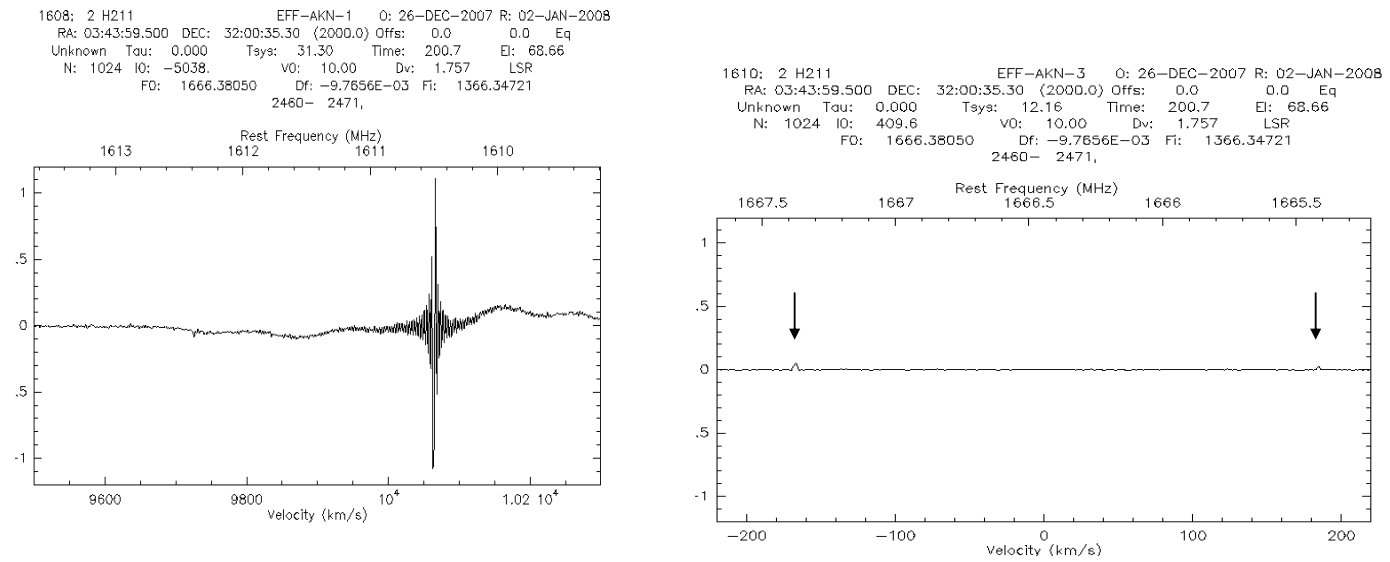

Fig. 3. Interference received from a low earth orbit mobile phone satellite (right) and a typical cosmic radio source (two small bumps in the left panel) at $1610 \mathrm{GHz}$ (diagrams courtesy of A. Kraus).

A few numbers may serve to illustrate the magnitude of the quantities and of the achieved sensitivities: one Jy is the spectral flux density that one would receive on the ground from a $1 \mathrm{~W}$ e.i.r.p IMT-2000 mobile phone operating at $2.1 \mathrm{GHz}$ and orbiting the earth at a distance of $8.867 \times 10^{5} \mathrm{~km}$, more than twice the distance to the moon! The main beam sensitivity of a large radio telescope at that frequency can exceed $50 \mathrm{dBi}$. If we assume a $10 \mathrm{MHz}$ detection bandwidth, the $100 \mathrm{~m}$ Effelsberg radio telescope is capable of detecting such a $1 \mathrm{~W}$ e.i.r.p. source at a distance of $6.9 \times 10^{7} \mathrm{~km}$ after $2000 \mathrm{~s}$. In this case we receive a spectral flux density of $0.17 \mathrm{mJy}$, which is a typical strength of the signals received from distant cosmic sources. Figure 3 illustrates the difference between the signals strengths of interference and a cosmic source.

The interference source was one of the IRIDIUM global mobile phone satellites at a distance of $1500 \mathrm{~km}$. They employ a spot beam with a peak e.i.r.p. of $12.5 \mathrm{~W}$ and our measurements have shown that their seventh-order intermodulation products with an e.i.r.p. of $1 \mathrm{~mW}$ (ECC Rep. 171, 2011; ECC Doc. SE40(09)092, 2009) are causing the interference even when the satellite is not crossing the telescope beam.

Radio observatories are also vulnerable to regular and irregular impulsive interference. When they observe Pulsars, a class of radio sources that emits short radio pulses with periods ranging from $1.5 \mathrm{~ms}$ to more than eight seconds, they employ sensitive high time resolution detectors. Simple electric cattle fences in about two $\mathrm{km}$ distance from the telescope have frequently been detected as regular pulsed interference.

It becomes clear, that local radio emissions, even those that are considered weak by normal standards can become the source of severe interference for a radio observatory. This is particularly true, when the emission occurs within the radio astronomical bands. Frequency regulators have recognised the problem and implemented a complex set of protection measures for radio astronomy, based on extensive studies that investigated the compatibility of radio astronomy with active radio services.

\section{Industrial interference}

The ITU came into being because there was a need to coordinate the frequencies and locations of radio transmitters world-wide, for radio waves can travel far and by themselves do not respect conventions such as national boundaries. Hence the focus on ITU regulations is on transmissions intended for communication and information transfer in the widest possible sense. That includes not only broadcasting, radio amateurs, location tracking (radar), but also remote sensing and radio astronomy.

ITU regulations however do not deal extensively with radiation emanating from technical equipment that is not intended for communication. Even in the times before the ubiquitous use of digital data processing and fast power switching gear, the emissions from industrial and consumer electrical installations were already present and came under the headings of "Electromagnetic Compatibilty (EMC)", and "Electromagnetic Interference (EMI)", in contrast to "radio interference" from actual radio transmitters. In the past EMI affected mainly the spectrum below $100 \mathrm{MHz}$. Figure 4, taken from an ITU source (ITU-R P. 372-9, 2007) reflects this. The earth's ionosphere effectively blocks the reception of radio waves from non-terrestrial sources below $30 \mathrm{MHz}$. One may note, that trace $\mathrm{E}$ of Fig. 4 indicates that the contribution of EMI to the radio background in typical cities exceeds that of "galactic radio noise" by about $20 \mathrm{~dB}$, converted in radio astronomical units we are talking of an ambient noise floor of several hundred Jy. This may serve to illustrate the reason why radio observatories are built in remote areas where manmade noise is expected to be less than the strength of cosmic radio signals (Fig. 4, trace D). The difference was mainly caused by the attenuation of interference emissions coming 


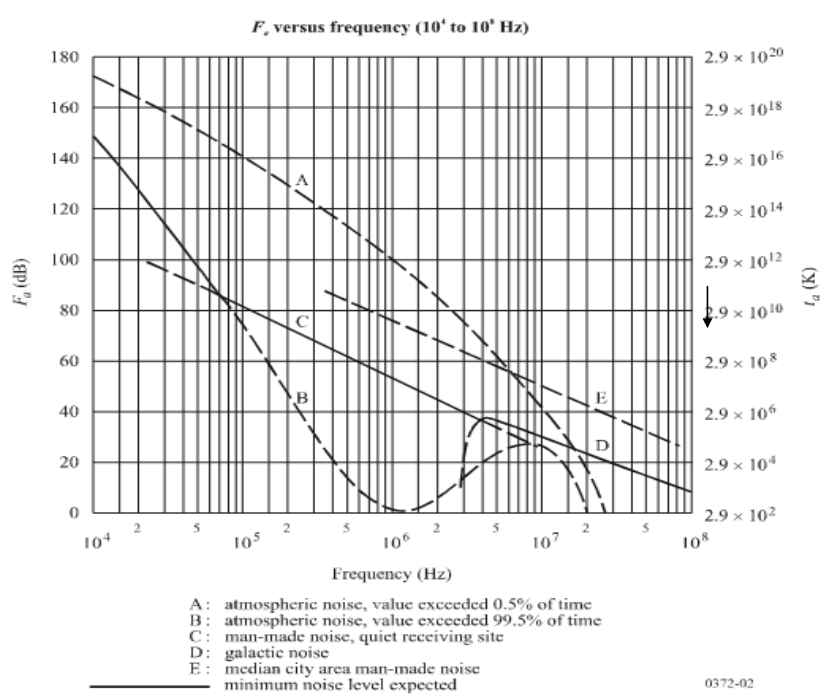

Fig. 4. Contributions to the radio noise environment (from ITU-R P. 372-9, 2007).

from localised concentrations of fixed installations in cities and industrial areas by their distance to the radio observatory and the terrain.

However with the increased use of high speed digital devices contained in license exempt and uncontrolled and often mobile consumer electronics as well as the spread of efficient high speed switching gear in consumer and industrial power conversion equipment, ranging from simple switch mode power supplies for a laptop to MW-size generators and converters in wind power plant, brings the sources of industrial interference closer to the radio observatories. Not only that, their interference spectrum will also be broader as it is naturally related to the rise and fall times of the digital switching used and these have become much faster with the recent advances in semiconductor electronics.

Standards are needed to limit the emission from such devices in order to suppress interference to communication equipment and also to other sensitive electrical equipment. These have been devised by CISPR $^{2}$ for a range of equipment separated into different categories and classes according to their use and construction. A list is given in the CISPR guide of the IEC (IEC, 2007). There two main classes:

1. equipment intended for use in a commercial and/or light industrial environment,

2. equipment intended for domestic use.

Class B equipment has to fulfil more stringent requirements than that from class A.

\footnotetext{
${ }^{2}$ Comité International Spécial des Perturbations Radioélectriques, the "special international committee on radio interference" is part of the international electro-technical commission (IEC).
}

CISPR limits are defined for the interferer. Time-averages and spectral properties are no always specified.

\section{Compatibility}

Many different interference scenarios that we did not have in the past have started to appear. The domestic use of computers, micro wave ovens, energy saving electric lights, has become common not only in the city but also in the rural environment close to radio observatories. The new scenario requires a detailed study of the impact of new industrial devices on radio astronomical measurements. In this paper we will look at the example of industrial switch-mode power converters in the $\mathrm{kW}$ to MW range which are often found in association with wind power generators or solar power stations. Like radio observatories, these are also erected in remote areas in order to avoid densely populated areas ${ }^{3}$.

The radio disturbance characteristics and limits are given as field strengths at a distance of $30 \mathrm{~m}$ in table 17 of the European standard CISPR-11 (= EN DIN 55011 in Germany) for such devices belonging to group 1 class A:

$30 \mathrm{~dB} \mu \mathrm{V} \mathrm{m}^{-1}$ for $f<230 \mathrm{MHz}$ and $120 \mathrm{kHz}$ measurement bandwidth

$37 \mathrm{~dB} \mu \mathrm{V} \mathrm{m}^{-1}$ for $230 \mathrm{MHz}<f<1 \mathrm{GHz}$ and $1 \mathrm{MHz}$ measurement bandwidth

No limits are prescribed for $f>1 \mathrm{GHz}$ and we will therefore continue to use the limit of

$$
37 \mathrm{~dB} \mu \mathrm{V} \mathrm{m}^{-1} \text { for all } f>1 \mathrm{GHz}
$$

A quasi peak detector is to be used for the measurements and no further specification of the spectrum apart from measurement bandwidth has been made. Many devices may emit narrow band radiation in the form of harmonics of their principal operating frequency, with a spacing that corresponds to the fundamental clock frequency. Radio astronomical bands are several $\mathrm{MHz}$ to several tens of $\mathrm{MHz}$ wide and powerful switches with switching rates of the order of a few ten $\mathrm{kHz}$ will create many harmonics within a radio astronomical band, creating an interference that often approximates a broad band signal.

However the increasing complexity and sophistication of the control and electronics of such devices, may also result in broad band interference, e.g. when switching frequencies and duty cycles vary on short timescales. Specifying peak or quasi-peak values only, makes the duration of the interference uncertain, it could last only for a few ms or for many

\footnotetext{
${ }^{3}$ This document should however not be interpreted as one of fundamental opposition to modern electronics or to the measures to save energy and energy generation from renewable sources. On the contrary, scientists see that as an important contribution to averting a climatic catastrophe and to secure a sustainable level of human civilisation.
} 


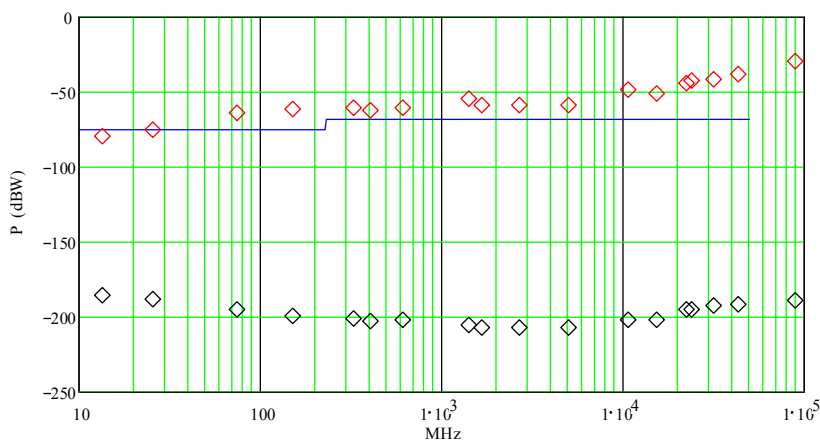

Fig. 5. Emission and reception power limits as specified by CISPR11 and ITU-R RA 769. The blue trace is the e.i.r.p. per measurement channel as given by CISPR-11, the red diamonds indicate the maximum broad band emission within the allocated radio astronomy band and the black diamonds show the reception limits from ITU R-RA 769.

days. This will affect radiometric measurements differently (see Eq. 1). The unspecific nature of the spectral and temporal characteristics of potential interferers makes generic compatibility assessments uncertain. In compatibility studies for passive services one is forced to err on the safe side and that may impose a greater constraint on emission levels than actually needed if a more informative specification of the interferer emissions were available.

It is useful to calculate the equivalent e.i.r.p. of the interference source for the given field strengths $E$ and distances $d$ :

$P_{\text {eirp }}=E+20 \log (d)-134.8$

where the power is given in $\mathrm{dB}(\mathrm{W})$, the electrical field strength $E$ in $\mathrm{dB}\left(\mu \mathrm{V} \mathrm{m}^{-1}\right)$ and the distance $d$ from the source in $\mathrm{m}$. For the listed values of 30 and $37 \mathrm{~dB}\left(\mu \mathrm{V} \mathrm{m}^{-1}\right)$ we obtain emission e.i.r.p of -75 and $-68 \mathrm{~dB}(\mathrm{~W})$, emission powers that are only fractions of a $\mu \mathrm{W}$. This has to be contrasted with the $60 \mathrm{dBW}$ power level that is going to be processed in the switching converter. Although one knows that e.g. the harmonics generated by fast switching decay rapidly with frequency, it is also conceivable that meeting a target of $135 \mathrm{~dB}$ for the suppression of high frequency harmonics can be difficult.

ITU RA-769 gives in its Table 1 the threshold power $\Delta P_{\mathrm{H}}$ in $\mathrm{dB}(\mathrm{W})$ for a radio astronomical receiver that would be considered as detrimental interference. The received power is meant to be averaged over the total allocated bandwidth for a specified integration time. Threshold values scale according to Eq. (1) for different receiver bandwidths and averaging times. Figure 5 shows the emission and reception thresholds in one graph: the difference between the emission and reception limits constitutes the minimum coupling loss (MCL) that is required to shield the radio receiver from the interferer. It is of the order of 140-160 dB and Fig. 6 shows it for narrow and broad band emissions as a function of frequency.

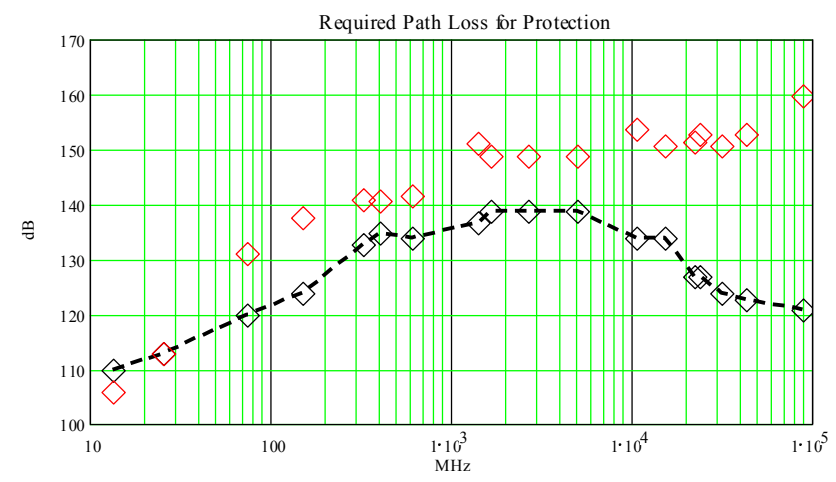

Fig. 6. MCL values for the protection of radio astronomy from devices fulfilling the CISPR-11 standard. Black line: Narrow band emission spectrum with one line per radio astronomical band, red diamonds: MCL for broad band emission still within the CISPR-11 limits.

The effects of uncertainty about spectral properties result in significant variation of the MCL at frequencies above $1 \mathrm{GHz}$ and even more so above $10 \mathrm{GHz}$. For large power plants high frequency emissions in the $10 \mathrm{GHz}$ range are not expected to be a problem, but they can be important for other types of devices that have emission standards that are similarly defined but where there is a potential of significant broad band emissions in the $\mathrm{GHz}$ range.

Assuming unobstructed line of sight propagation without atmospheric absorption one can find the minimum distance required from

$d=10^{\frac{\mathrm{MCL}-20 \log (f)-92.5-A_{\mathrm{ht}}}{20}}$

given in $\mathrm{km}$, with the minimum coupling loss in $\mathrm{dB}$, the frequency in $\mathrm{MHz}$ and $A_{\mathrm{ht}}$ a correction factor for the absorption by ground clutter that depends on terrain properties and height of the transmitter. It ranges from $0 \mathrm{~dB}$ at heights exceeding $20 \mathrm{~m}$ to roughly $20 \mathrm{~dB}$ for ground level emissions (ITU-R P.452, 2005). Figure 7 shows the estimated separation distances as a function of frequency.

It is evident that electronic equipment complying with the industrial standard CISPR-11 has the potential to interfere on VHF and UHF bands over distances significantly greater than the line of sight horizon (typically $25 \mathrm{~km}$ ) for the radio telescope (nominal height $50 \mathrm{~m}$ ) and any ground level site of electronic equipment. However, the curvature of the earth and local topography will often provide additional shielding. A case by case study is required when electronic devices are to be operated within the line of sight of a radio telescope. As a result one may have to specify stricter limits than CISPR11 , in some locations but not in others.

An example of how the terrain affects the local attenuation is given in Fig. 7 for a solar power station close to a projected radio astronomical observatory in South Africa. The terrain is flat and there are large areas over distances of more than $30-40 \mathrm{~km}$ for which emissions from a power plant may 


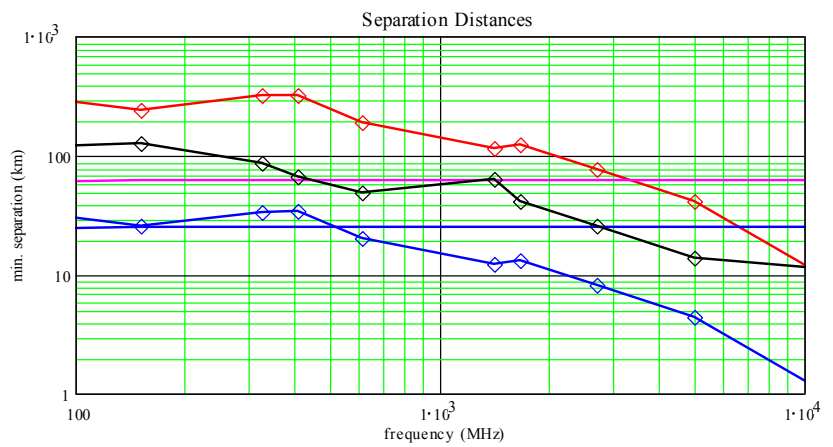

Fig. 7. Separation distances between CISPR-11 certified devices and radio astronomical stations. The red line shows the unobstructed free-space separation distance for equipment complying with CISPR-11 (EN55011) having only one narrow emission line within the radio astronomical band. The blue line with diamonds shows the separation distance for narrow band emitters on the ground (clutter absorption) and the black line that for broad band emitters. The $25 \mathrm{~km}$ line of sight horizon of the radio telescope and the $61 \mathrm{~km}$ visibility horizon for the hub of a windmill at a height of $100 \mathrm{~m}$ are shown by horizontal lines (blue and mauve).

degrade the measurements if their emission levels actually reach the limits specified in the CISPR-11 standard.

\section{Specific mitigations and their assessment}

Operational factors, such as intermittent activity, improved shielding or design that has inherently lower emissions (steep spectrum with only a few lines) will mitigate the impact the emitted interference. However these factors need to be carefully assessed and verified before they can enter a compatibility study. Standard measurement techniques, such as those prescribed in the CISPR standards may not be sensitive enough to verify the emission levels of $20-40 \mathrm{~dB}$ below the norm which may be needed for compatibility in some situations. An ordinary spectrometer, even combined with a low-noise frontend will usually not show any interference on such low levels as its intrinsic noise level is too high, especially when the normally short effective integration times are considered. But radiometric measurements are always possible if the location is radio-quiet. For that one would need to subtract the integrated linear scaled power spectrum of the background (device turned off) from the one taken with the device in operation. Equation (1) may be used to calculate the required averaging times for a desired sensitivity.

Separation distances can change significantly and in a nonlinear way with mitigation, but terrain effects clearly dominate as can be seen in Fig. 8. The graph illustrates the example of interference on the radio astronomical band 1400 $1427 \mathrm{MHz}$ where according to RR footnote 5.340 "no emissions are permitted". For devices on the ground, we find that narrow band rfi can be tolerated for distances greater than

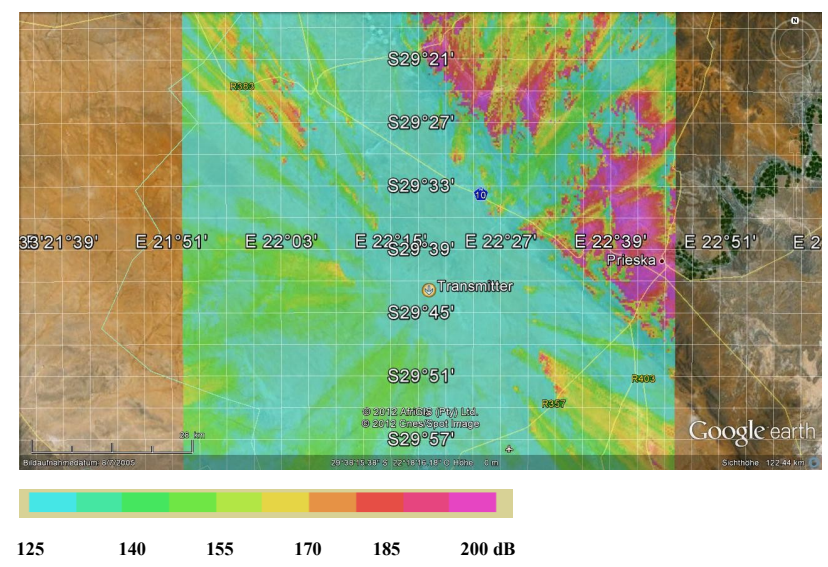

Fig. 8. Path loss map calculated for a South African site (indicated by "Transmitter") for emissions at $610 \mathrm{MHz}$ and an emission height of $5 \mathrm{~m}$ using "Pathprofile" (CRAF, 2012). The MCL for narrow band emissions at that frequency is $134 \mathrm{~dB}$ and for broad band emissions it is $141 \mathrm{~dB}$. All green areas are unsuitable as sites for a power station that will have emissions around the CISPR-11 limits.

$12.3 \mathrm{~km}$ and broad band rfi would require a separation of $34.8 \mathrm{~km}$. A $20 \mathrm{~dB}$ mitigation through improved shielding, intrinsically lower emissions or a $1 \%$ percent duty cycle will reduce the distance to $1.2 \mathrm{~km}$ for narrow band and $6.2 \mathrm{~km}$ for broad band rfi. In order to have an unrestricted deployment as close as $100 \mathrm{~m}$ from the antenna, one would require a reduction of more than $46 \mathrm{~dB}$ for broad band emissions down to a level of $-114 \mathrm{~dB}(\mathrm{~W} / \mathrm{MHz})$. Note that within the EU, a limit of $-120 \mathrm{~dB}(\mathrm{~W} / \mathrm{MHz})$ is specified for license-free UWB devices for frequencies below $1.6 \mathrm{GHz}$ (ECC Decision (06)04, 2007), which is $52 \mathrm{~dB}$ more stringent than CISPR-11! For installations in the hub of a wind power generator at a height of $100 \mathrm{~m}$, and a distance of $2 \mathrm{~km}$ the requirements are similar: $36 \mathrm{~dB}$ reductions are needed in the case of narrow-band emission and a $50 \mathrm{~dB}$ reduction is required for broad band emissions (MCL of $150 \mathrm{~dB}$ !). It is conceivable that an ACgenerator combined with filtered supply cables down to a shielded power converter on the ground may even achieve such a low level of emissions, however the verification will become quite difficult.

\section{Aggregation of interference}

Depending on the type of equipment, we may see a large deployment of devices over the area around the observatory and even if one can exclude or mitigate the effects of potential interferers close to the radio astronomy site, their aggregate effect may raise the ambient noise levels as seen for the general noise background in Fig. 4. The study of aggregation will provide another set of threshold levels, usually derived for an idealised situation where a large number of trials are made with devices placed randomly over an area centred 


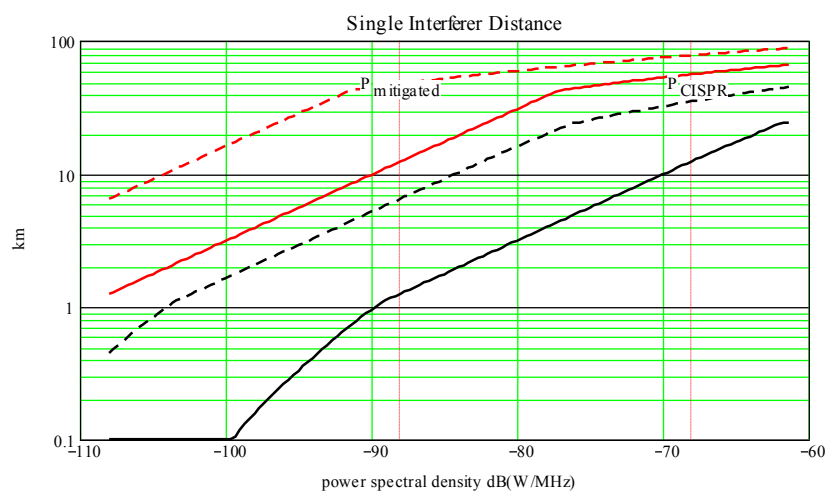

Fig. 9. Single interferer separation distances calculated for $f=$ $1.41 \mathrm{GHz}$ using the propagation model of ITU-R P.452 for flat terrain, but including the effects of ground clutter and curvature of the earth. Black solid line: narrow band emission at a height of 1 $\mathrm{m}$, black dashed line: broad band emission. Red solid line: narrow band emission at a height of $100 \mathrm{~m}$. Red dashed line: broad band emission at a height of $100 \mathrm{~m}$.

around the receiver and at different heights if so required for a realistic modelling of the deployment scenario. Their average distribution is set to match the average deployment values and emission levels. One then calculates the sum of their signal powers using known propagation mechanisms to obtain the received interference power for each trial. The results statistically evaluated and plotted in histograms like the one shown in Fig. 9 for the $1.4 \mathrm{GHz}$ scenario outlined above. For an assumed deployment density of $1 \mathrm{~km}^{-2}$ and two possible operational heights we find that the average received power level amounts to $-177 \mathrm{~dB}(\mathrm{~W})$, exceeding the threshold ITUR 769 threshold of $-204 \mathrm{~dB}(\mathrm{~W})$ by $27 \mathrm{~dB}$, the median is at $-181 \mathrm{~dB}(\mathrm{~W})$ and in $2 \%$ of all cases, the threshold was exceeded by more than $36 \mathrm{~dB}$.

Although transmitter emissions are not permitted in the band we have just studied, radiation by electronic devices cannot be totally avoided and, an aggregation study may indicate the probability of interference when the deployment density of devices is known and their emissions are already reduced by technical or operational means. Different interference probabilities are needed depending on the allocation and protection status of the band. For shared bands (Radio Regulations FN 5.149) a $2 \%$ interference probability is accepted as a practical measure, however purely passive bands require much lower interference probabilities derivable from the threshold values and the noise statistics. In addition one has to be aware of and take precautions for the fact, that a prediction of deployment characteristics for any new device is always conjectural and the actual level of interference from widely distributed license-free devices can only be known when deployment numbers have stabilised.

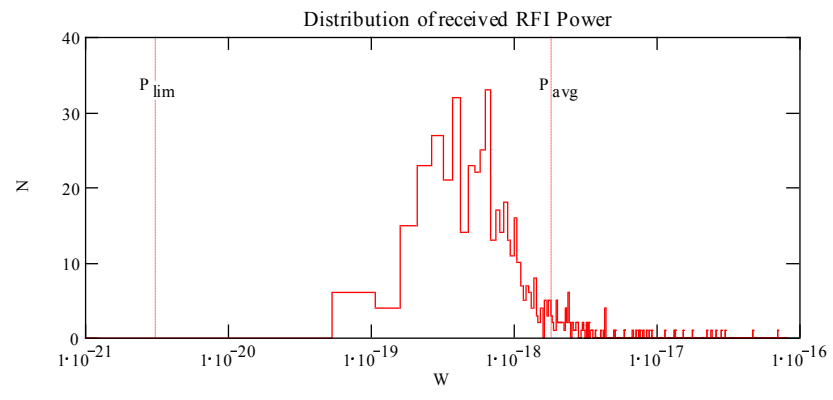

Fig. 10. Histogram of received power levels for 500 trials with random placements of narrow band CISPR-11 certified devices at $1.4 \mathrm{GHz}$ within a range of $92 \mathrm{~km}$ from the radio telescope. The contributions from two operational heights $(1 \mathrm{~m}$ and $100 \mathrm{~m})$ were averaged for each of the 84 placements per trial for a deployment density of $1 \mathrm{~km}^{-2}$. Average signal values are $27 \mathrm{~dB}$ above the ITU-R RA 769 limits designated by $P_{\text {lim }}$.

\section{Conclusions}

In the past, radio observatories and other passive services have had the opportunity of choosing remote, radio quiet rural locations which had a significantly lower level of manmade interference. The proliferation of fast switching power equipment and other digital technologies is a necessary step in the direction of greater energy conservation and use of renewable energy sources. This can however result in severe interference problems for passive services such as radio astronomy if compatibility questions not addressed at an early stage. Regulatory administrations have recognised the problem for unlicensed and uncontrolled consumer communications equipment, but this is not the case for industrial devices.

If a radio astronomical observation is obliterated by interference, the information that could be obtained is lost. It is irrelevant if the origin of rfi is from industrial or communication equipment, the distinction becomes more and more artificial with the progress of digital technology and it is now time to harmonise the emission standards for both the communications and the industrial realm.

Acknowledgements. The author likes to thank Mike Willis for the use of his pathprofile program and Alex Kraus, Benjamin Winkel, Harry Smith and Reinhard Keller for support and discussions.

\section{References}

Cane, H. V.: MNRAS, 189, 465, 1979.

CISPR Guide, IEC, Geneva, available at: http://www.iec.ch/emc/ iec_emc/iec_emc_players_cispr.htm, 2010.

CRAF Newsletter 25, 7, available at: http://www.craf.eu/nwsl25. pdf, 2012.

Dicke, R. H.: The measurement of thermal radiation at Microwave frequencies, Rev. of Sci. Instr., 268, 17, 1946. 
ECC Decision (06)04 on the harmonised conditions for devices using Ultra-Wideband (UWB) technology in bands below 10.6 GHz, Constanta, 2007.

ECC Doc. SE40(09)092: Assessment of Intermodulation products for the satellite transmitters and the victim receivers at the radio astronomy station, Copenhagen, 2009.

ECC Rep. 171: Impact of unwanted emissions of IRIDIUM satellites on radio astrononomy operations in the band 1610.6-1613.8 MHz, Tallinn, 2011.
Ellington IEEE Trans on Antennas and Propagation, 53, No. 8, 2480, 2005.

ITU-R RA. 769-2: Protection criteria used for radio astronomical measurements, ITU, Geneva, 2003.

ITU-R P. 372-2: Radio noise, ITU, Geneva, 2007.

ITU-R P. 452 Prediction procedure for the evaluation of microwave interference between stations on the surface of the earth at frequencies above $0.7 \mathrm{GHz}$, ITU, Geneva, 2005.

Radio Regulations, ITU, Geneva, 2012. 